\title{
Crystallography of Functional Materials
}

\author{
Winnie Wong-Ng ${ }^{1, *}$ (1) and Claudia J. Rawn ${ }^{2}$ \\ 1 Materials Measurement Science Division, National Institute of Standards and Technology, \\ Gaithersburg, MD 20899, USA \\ 2 Department of Materials Science and Engineering, University of Tennessee, Knoxville, TN 37996, USA; \\ crawn@utk.edu \\ * Correspondence: Winnie.wong-ng@nist.gov; Tel.: +1-301-975-5791
}

Received: 13 September 2017; Accepted: 13 September 2017; Published: 15 September 2017

\begin{abstract}
The goal of this special issue is to obtain new insights into the roles of crystallography in functional materials. This special issue consists of eight papers illustrating the structure and property relationships, as well as applications of selected classes of materials that deal with various aspects of functional materials, ranging from battery, magnetic, photocatalysis, and waveguide materials, to luminescent metal-organic frameworks and borates, semiconductors, and inorganic electrides. This issue provides further evidence of the importance of crystallography in understanding and improving various properties of functional materials, whether they are single crystals, bulk polycrystalline materials, or thin films.
\end{abstract}

Keywords: crystallography; functional materials; structure/property relationships

\section{Introduction}

Functional materials, which possess specific properties and functions, form the basis for a wide range of technologies, including electronics, information storage and retrieval, communication, biotechnology, aerospace, defense, environmental, energy, medical, and consumer products. A partial list of the special chemical, electrical, optical, and physical properties of these materials includes magnetism, semiconducting behavior, ferroelectricity, piezoelectricity, ferromagnetism, ionic conductivity, superconductivity, photocatalytic behavior, adsorption behavior, and giant magneto-resistance, with resulting specific applications in microelectronic devices and data storage, sensing, energy storage, hydrogen storage, thermoelectric waste heat conversion, solar cells, fuel cells, batteries, and other energy harvesting and conversion applications. Functional materials of interest cover different classes of solid-state materials ranging from semiconductors to polymers, from molecular crystals to nanoparticles. The development and fundamental understanding of the properties of functional materials are central to technological needs, and are at the forefront of materials research.

The field of crystallography provides the foundation of the structure-properties relationship. It bridges the fundamental understanding of materials with their applications. As functional materials play a critical role in our modern society and future economic, physical, and social success, the underlying crystallographic information for these materials is of utmost importance for many research communities.

To date, a number of journals have been devoted to the important subject of functional materials including Journal of Functional Materials, Functional Materials Letters, Advanced Functional Materials, and Journal of Functional Materials and Devices. However, despite the diverse subjects covered in these journals, there has not been a focus on the importance of the relationships between crystallography and representative functional properties. Therefore, the journal "Crystals" provides an important 
venue (this special issue) for authors and readers to recognize, understand, and appreciate the impact of these subjects.

In the current millennium, functional materials will play an increasingly vital role in economic growth and quality of life. The goal of this special issue is to cover the latest developments in the cross disciplinary fields of structure science and functional materials, to provide structure and property relationships including but not limited to phase transformations, local structures, modular and disordered structures, and defects. The forms of materials of interest include single crystals, bulk ceramics, amorphous solids, liquid crystals, thin films, and nanostructures. In situ and in operando crystallography and high-pressure crystallography are also important areas in this context. In the following, we will comment on the essence of the eight chapters in this issue.

\section{Representative Functional Materials}

\subsection{Ge-Waveguide Materials}

An optical waveguide is a physical structure that guides electromagnetic waves in the optical spectrum. Common types of optical waveguides include optical fiber and rectangular waveguides. Optical waveguides are used as components in integrated optical circuits or as the transmission medium in local and long haul optical communication systems. These waveguides in general are classified according to their geometry (planar, strip, or fiber waveguides), mode structure (single-mode, multi-mode), refractive index distribution (step or gradient index), and material (glass, polymer, semiconductor).

Qi et al. [1] reported that tensile-strained Ge has been investigated as a possible laser material due to two main factors, (1) nearly-direct band gap and (2) its compatibility with conventional silicon integrated circuit fabrication. However, despite the multitude of benefits, problems remain due to the fact that lasers using tensile-strained Ge show a high threshold current density and short lifetime. To understand the origins of these problems, the authors used the combined techniques of Raman spectroscopy and time-dependent diffraction techniques such as high-resolution transmission electron microscopy (HRTEM). Real-time degradation in tensile-strained Ge was observed, and a network of defects was observed to propagate with a speed and density correlated with the local strain. Therefore, highly tensile-strained Ge lasers (which can lead to the formation of dislocations and stacking faults) indeed have a short lifetime, and they are better suited for low-energy devices than they are for lasers.

\subsection{Advanced Li-Ion Battery materials}

Lithium-ion batteries, invented in the late 1970s and early 1980s and commercialized in the 1990s, generate electrical currents when lithium ions travel between two electrodes. Light but powerful, they have transformed portable electronics. Their use in electric cars, however, is recent. When Tesla Motors [2] introduced one of the first lithium-ion-powered electric cars in 2008, it could go $250 \mathrm{~m}$ on a charge, but the vehicle cost over $\$ 100,000$, in large part because the batteries are expensive [3]. To cut costs, the lithium-ion-powered electric cars made today by companies such as Nissan and GM use small battery packs with a range of less than $100 \mathrm{~m}$. While countless breakthroughs have been announced over the last decade, these advances failed to translate into commercial batteries. One difficulty about developing better batteries is that the Li-ion battery technology is still poorly understood, including the crystallography of the important cathode oxide materials.

Bendersky et al. [4] investigated the crystallography and growth of epitaxial oxide films for an in-depth understanding of the cathode materials used in advanced Li-ion batteries. Details of film growth, including the identity of each of the layer forms, are important to understand the charge and discharge mechanism. High-resolution Transmission Electron Microscopy (HRTEM) is by far the most efficient and sophisticated technique for identifying various layers of film growth and their crystallographic relationships. Bendersky et al. [4] studied a number of Li-containing oxide compositions representing different functions. Results indicated that epitaxial film growth is possible 
for all films, with the growth following the predicted cube-on-cube orientation relationship between the cubic and pseudo-cubic oxygen sublattices of a film and a substrate. These results provide new insights into electrochemical processes occurring in the bulk and at interfaces.

\subsection{Luminescence Properties of Borates}

Inorganic light emitting diode (LED) materials are one of the major research areas within solid-state luminescence. Of special interest is generating white LED light and one approach to achieve this uses an inorganic phosphor material to convert near-UV radiation, emitted from a LED, to white light. In the paper by Zhao et al. [5], the authors report on the crystal structure of $\mathrm{Na}_{3} \mathrm{Sm}\left(\mathrm{BO}_{3}\right)_{2}$ as a potential crystalline host material. Recently, Wang et al. [6] reported and characterized the $\mathrm{Na}_{3} \mathrm{Sm}\left(\mathrm{BO}_{3}\right)_{2}$ compound using X-ray powder diffraction, while the work presented in this special volume by Zhao et al. demonstrates the growth of a single crystal of $\mathrm{Na}_{3} \mathrm{Sm}\left(\mathrm{BO}_{3}\right)_{2}$, using a high temperature flux method. Zhao et al. [5] studied the atomic structure via single crystal X-ray diffraction and the thermal stability of the compound using TG/DTA. The paper also presents the ultraviolet-visible (UV-vis) absorption spectrum, the excitation and emission spectra, decay time, and location of the $\mathrm{Na}_{3} \mathrm{Sm}\left(\mathrm{BO}_{3}\right)_{2}$ phosphor on the Commission International de l'Éclairage (CIE) chromaticity index. The results of their structural and luminescent properties characterization lead the authors to conclude that $\mathrm{Na}_{3} \mathrm{Sm}\left(\mathrm{BO}_{3}\right)_{2}$ could potentially be used as an orange-red phosphor under near-UV light excitation.

\subsection{Layered Magnetic Metal Halides}

The review paper by McGuire [7] examines the crystal structure and magnetic properties of $\mathrm{MX}_{2}$ and $\mathrm{MX}_{3}$ compounds where $\mathrm{M}$ is a transition metal with partially filled d-shells $(\mathrm{M}=\mathrm{Ti}, \mathrm{V}, \mathrm{Cr}, \mathrm{Mn}, \mathrm{Fe}$, $\mathrm{Co}, \mathrm{Ni}, \mathrm{Zr}, \mathrm{Mo}, \mathrm{Tc}, \mathrm{Ru}, \mathrm{Rh}$, and $\mathrm{Ir}$ ) and $\mathrm{X}$ is either $\mathrm{Cl}, \mathrm{Br}$, or I. When $\mathrm{M}=\mathrm{Ti}, \mathrm{V}, \mathrm{Mn}, \mathrm{Fe}, \mathrm{Co}$, or Ni the $\mathrm{MX}{ }_{2}$ compound crystallizes in either the $\mathrm{CdI}_{2}$ or $\mathrm{CdCl}_{2}$ structure types. However, when $\mathrm{M}=\mathrm{Zr}$ and $\mathrm{X}=\mathrm{Cl}$, the compound crystallizes with the $\mathrm{MoS}_{2}$ structure type; when $\mathrm{X}=\mathrm{I}$, it can crystalize as either the $\mathrm{MoTe}_{2}$ or $\mathrm{WTe}_{2}$ structure types. The $\mathrm{CdI}_{2}$ and $\mathrm{CdCl}_{2}$ structure types have in common edge sharing octahedra, creating triangular nets of cations resulting in $\mathrm{MX}_{2}$ layers perpendicular to the $c$-direction, and are distinguished by layering in the $c$-direction. The $\mathrm{MX}_{3}$ compounds predominately crystalize in the $\mathrm{BiI}_{3}$ or $\mathrm{AlCl}_{3}$ structure types and again have edge sharing octahedra, but for these structure types a hexagonal net of cations results, and there are many variants of the stacking sequences resulting from the weak van der Waals interactions between the layers. Despite the common building blocks the $\mathrm{MX}_{2}$ and $\mathrm{MX}_{3}$ compounds have a wide variety of interesting magnetic structures and behaviors.

\subsection{Structure and Luminescent Properties of Metal-Organic Frameworks (MOF)}

Metal-organic frameworks (MOFs) are promising multifunctional luminescent materials because of their inherent advantages of containing both organic linkers and inorganic metal ions, as well as tailorability in terms of structure, dimension, size, and shape. Furthermore, the metal centers, organic linkers, metal-organic charge transfer, and guest molecules within porous MOFs all can potentially generate luminescence. Such uniqueness can allow us to generate luminescent MOF materials with systematically varied luminescence properties, which are crucial for the lighting, display, and optical devices. Although the development of luminescent MOFs is still in its infancy, the currently available results have unambiguously demonstrated that the design and construction of MOFs for luminescent functionality is an active field, and hundreds of papers published in the last few years indicate that this interest is increasing.

The chapter by Barsukova et al. [8] outlines four new Zn(II) and Cd(II)-based flexible MOF materials, with bis(imidazole-1-yl) alkane as the ligands: their synthesis, structural characterization, and solid-state luminescence measurements. The authors concluded that the structure of these compounds, particularly the length of the flexible linkers, has a great influence on the optical properties. 


\subsection{Semiconductor Porphyrins}

Porphyrins are a group of heterocyclic macrocycle organic compounds, composed of four modified pyrrole subunits interconnected at their $\alpha$ carbon atoms via methine bridges $(=\mathrm{CH}-)$. The parent porphyrin is porphin, and substituted porphines are called porphyrins. The main functions of porphyrins, in addition to supporting aerobic life, include a variety of applications based on their optical, semiconducting, and redox properties. They can perform electronic functions in devices ranging from diodes to rectifiers, wires, and capacitors, and as materials in electronic or magnetic memory.

The crystallographic features of various porphyrins are fascinating and the various features govern the subsequent electronic properties. The structure of the material, particularly the planarity of the macrocycle and the conjugation of $p i$-bonding through the proximity of adjacent macrocycles or the interconnection of macrocycles via appropriate covalently-bonded ligands, play a pivotal role in the solid state semiconducting properties of porphyrins.

Cook et al. [9] reviewed the structure of the parent porphyrin compounds, the peripheral substituents, the metalation of the macrocycle core, the role of covalent inter-molecular bonding, and the electronic properties as related to structure. Various factors, important in optimizing porphyrin semiconducting properties, were summarized and discussed at the molecular level, and in single crystals, thin films, and bulk polycrystalline materials. Finally, a number of structural examples of complex multiporphyrins were illustrated, including bridged multiporphyrins, confacial dimers, directly linked porphyrins, multiple-linked structures, self-assembled arrays, and metal-bridged arrays.

\subsection{Photocatalysis Materials Constructed $U$ sing $\alpha-U_{3} O_{8}$ Types of Layers}

The origins of a hydrogen energy economy go back to the 1920s, but it was not until the 1970s, an era where several energy/oil crises occurred, when the idea of using hydrogen to replace fossil fuels took traction and many scientists and engineers took up the challenges of producing and storing hydrogen. As the price of oil declined during the 1980s, so did the emphasis on finding alternatives. However, in the 2003 State of the Union Address President Bush stated, "A simple chemical reaction between hydrogen and oxygen generates energy, which can be used to power a car, producing only water, not exhaust fumes. With a new national commitment, our scientists and engineers will overcome obstacles to taking these cars from laboratory to showroom, so that the first car driven by a child born today could be powered by hydrogen, and pollution-free". With this statement, the hydrogen economy was back in focus, and research into the production and storage of hydrogen again briefly surged. In photoelectrochemical water splitting, the electricity used to produce the hydrogen is supplied by a renewable energy source (i.e., a photovoltaic system). In the paper presented by King et al. [10], the authors review crystal structures having photocatalytical properties. This paper focuses on a series of mixed-metal oxide structures, based on the stacking of $\alpha-\mathrm{U}_{3} \mathrm{O}_{8}$ type pentagonal bipyramid layers, with the general formula $\mathrm{A}^{m+}((n+1) / m) \mathrm{B}_{(3 n+1)} \mathrm{O}_{(8 n+3)}$, where the A cation can be Ag, Bi, Ca, Cu, Ce, Dy, $\mathrm{Eu}, \mathrm{Gd} \mathrm{K}, \mathrm{La}, \mathrm{Nd}, \mathrm{Pb}, \mathrm{Pr}, \mathrm{Sr}$, or $\mathrm{Y}$, the $\mathrm{B}$ cation is either $\mathrm{Nb}$ or $\mathrm{Ta}$, and $m=1-3$ and $n=1,1.5,2$. Some of the compounds from the series have phase transitions, associated with changes in temperature, composition, and chemical reactions, resulting in structural distortions that lower the symmetry of the crystal structure. Related to these structural distortions are electronic distortions that impact the efficiency of the photocatalysis process.

\subsection{Cationic Doping in $\left[\mathrm{Ca}_{24} \mathrm{Al}_{28} \mathrm{O}_{64}\right]^{4+}$ Clathrate Framework, an Electride}

The compound $\mathrm{Ca}_{12} \mathrm{Al}_{14} \mathrm{O}_{33}(\mathrm{Z}=2)$ is located close to the middle of the $\mathrm{CaO}-\mathrm{Al}_{2} \mathrm{O}_{3}$ binary phase diagram, a historic phase diagram used by many of the early ceramic industries and geologists, rich with intermediate compounds. $\mathrm{Ca}_{12} \mathrm{Al}_{14} \mathrm{O}_{33}$, or mayenite, has long been identified with various cement mixtures. The crystal structure of $\mathrm{Ca}_{12} \mathrm{Al}_{14} \mathrm{O}_{33}$ consists of a framework made up of the $\mathrm{Ca}$ and $\mathrm{Al}$ cations and 32 of the oxygen anions, forming a cage structure, with the remaining oxygen needed to 
charge balance the structure, occluded within the cage. Depending on the processing conditions, the occluded oxygen can be removed and replaced with other atoms, molecules, or electrons giving rise to a host of interesting properties including near metallic conduction. The ability for the electrons to remain in the structure at room temperature has earned the compound the distinction of being the first room-temperature inorganic electride [11]. Many researchers have tried doping the stoichiometric oxygen occupied structure with various cations to improve the resulting properties, but few have continued to study the change in the physical properties and electron behavior in the electride. The paper by Salasin and Rawn [12] provides a review of cationic doped isostructural compounds and various processing methods with the purpose of invigorating research into the effects that dopants can have on the density of states, localized electron behavior, structural distortions, and ultimately the resulting electronic properties.

\section{Conclusions}

The eight papers contained in this special issue on the crystallography of functional materials cover a large range of end applications, and each chapter works to link the various building blocks at the atomic scale to properties of interest including electrical, electrochemical, magnetic, and optical. Four of the eight papers worked to overview various structures and corresponding resulting properties, while the other four presented experimental results, two using X-ray diffraction and two using transmission electron microscopy (TEM). For the latter, Bendersky et al. [4] used high-resolution TEM to examine the cross sections of the Li-ion battery cathode materials of $\mathrm{LiCoO}_{2}, \mathrm{LiMnO}_{2}, \mathrm{Li}_{2} \mathrm{MnO}_{3}$, and $\mathrm{Li}_{1.2} \mathrm{Mn}_{0.55} \mathrm{Ni}_{0.15} \mathrm{Co}_{0.1} \mathrm{O}_{2}$ on $\mathrm{SrTiO}_{3}$ - or $\mathrm{SrRuO}_{3} / \mathrm{SrTiO}_{3}$-supporting substrates of different orientations. The experimental results indicated the near-coherent epitaxial growth of the cathode materials deposited by pulsed laser deposition (PLD). Three of the papers illustrate how processing conditions can be used to alter the structure and change the resulting properties. In the case of the Ge-waveguide material, the formation of dislocations and stacking faults, resulting from tensile-straining, imaged using TEM, illustrates the introduction of atomic scale defects that improve the resulting properties. In the case of the binary oxide compound $\mathrm{Ca}_{12} \mathrm{Al}_{14} \mathrm{O}_{33}$, firing in a reducing atmosphere results in the removal of occluded oxygen atoms, replacing them with other atoms, molecules, or electrons, where the latter can alter the conduction from highly resistive to near-metallic. King et al. [10] showed that mixed-metal oxide compounds, with the general formula $\mathrm{A}^{m+}{ }_{((n+1) / m)} \mathrm{B}_{(3 n+1)} \mathrm{O}_{(8 n+3)}$, phase transitions that occur from varying the temperature, the composition, or the chemical reactions, can result in structural and electronic distortions that impact the efficiency of the photocatalysis. Two of the papers reported the crystal structures of new compounds along with complementary property measurements. Zhao et al. [5] reported on a method of growing single crystals of $\mathrm{Na}_{3} \mathrm{Sm}\left(\mathrm{BO}_{3}\right)_{2}$, along with the crystal structure, thermal stability, ultraviolet-visible (UV-vis) absorption spectrum, excitation and emission spectra, decay time, and location of the phosphor on the Commission International de l'Éclairage (CIE) chromaticity index. The synthesis and structural characterization of four new metal-organic frameworks (MOFs), based on zinc and cadmium ions, were reported along with their excitation and emission luminescence spectra. Two papers overviewed crystallographic structures and various related features to properties with an eye on being able to exploit the features and develop new materials in the future with improved properties. The paper by McGuire [7] presented an overview of the crystal structures metal di- and tri-halides, calling attention to the triangular and honeycomb transition metal cation nets and stacking sequences, along with their magnetic structures and properties. The paper by Cook et al. [9] presented an overview of the crystallographic attributes of porphyrins with examples illustrating how the structural orientations of the porphyrin macrocycle and the inter-porphyrin covalent bonding present in multiporphyrins can influence the semiconducting properties.

Conflicts of Interest: The authors declare no conflict of interest. 


\section{References and Notes}

1. Qi, M.; O’Brien, W.A.; Stephenson, C.A.; Patel, V.; Cao, N.; Thibeault, B.J.; Schowalter, M.; Rosenauer, A.; Protasenko, V.; Xing, H.G.; et al. Extended Defect Propagation in Highly Tensile-Strained Ge Waveguides. Crystals 2017, 7, 157. [CrossRef]

2. The purpose of identifying the equipment and name of manufacturers in this article is to specify the experimental procedure or illustrate the purpose of project. Such identification does not imply recommendation or endorsement by the National Institute of Standards and Technology.

3. Tesla Debuts Electric Car For The Masses, CBC News, 2009-03-27. Available online: http:/ /www.cbc.ca/ news/technology/tesla-debuts-electric-car-for-the-masses-1.816478 (accessed on 20 July 2017).

4. Bendersky, L.A.; Tan, H.; Bharathi Karuppanan, K.; Li, Z.-P.; Johnston-Peck, A.C. Crystallography and Growth of Epitaxial Oxide Films for Fundamental Studies of Cathode Materials Used in Advanced Li-Ion Batteries. Crystals 2017, 7, 127. [CrossRef]

5. Zhao, D.; Ma, F.-X.; Nie, C.-K.; Wang, J.; Zhang, L.; Fan, Y. Structure Determination and Luminescent Property Studies of the Single Crystal $\mathrm{Na}_{3} \mathrm{Sm}\left(\mathrm{BO}_{3}\right)_{2}$. Crystals 2017, 7, 129. [CrossRef]

6. Wang, Z.X.; Li, H.K.; Cai, G.M.; Jin, Z.P. Synthesis, crystal structure, and thermal stability of new borates $\mathrm{Na}_{3} \mathrm{REB}_{2} \mathrm{O}_{6}(\mathrm{RE}=\mathrm{Pr}, \mathrm{Sm}, \mathrm{Eu})$. Powder Diff. 2016, 31, 110-117. [CrossRef]

7. McGuire, M.A. Crystal and Magnetic Structures in Layered, Transition Metal Dihalides and Trihalides. Crystals 2017, 7, 121. [CrossRef]

8. Barsukova, M.; Goncharova, T.; Samsonenko, D.; Dybtsev, D.; Potapov, A. Synthesis, Crystal Structure, and Luminescent Properties of New Zinc(II) and Cadmium(II) Metal-Organic Frameworks Based on Flexible Bis(imidazol-1-yl)alkane Ligands. Crystals 2016, 6, 132. [CrossRef]

9. Cook, L.P.; Brewer, G.; Wong-Ng, W. Structural Aspects of Porphyrins for Functional Materials Applications. Crystals 2017, 7, 223. [CrossRef]

10. King, N.; Boltersdorf, J.; Maggard, P.A.; Wong-Ng, W. Polymorphism and Structural Distortions of Mixed-Metal Oxide Photocatalysts Constructed with $\alpha-\mathrm{U}_{3} \mathrm{O}_{8}$ Types of Layers. Crystals 2017, 7, 145. [CrossRef]

11. Matsuishi, S.; Toda, Y.; Miyakawa, M.; Hayashi, K.; Kamiya, T.; Hirano, M.; Tanaka, I.; Hosono, H. High-Density Electron Anions in a Nanoporous Single Crystal: $\left[\mathrm{Ca}_{24} \mathrm{Al}_{28} \mathrm{O}_{64}\right]_{4+}(4 \mathrm{e}-)$. Science 2003, 301, 626. [CrossRef] [PubMed]

12. Salasin, J.R.; Rawn, C. Structure Property Relationships and Cationic Doping in $\left[\mathrm{Ca}_{24} \mathrm{Al}_{28} \mathrm{O}_{64}\right]^{4+}$ Framework: A Review. Crystals 2017, 7, 143. [CrossRef] 